\title{
PALLIATIVE CARE
}

\section{Benefits of Liverpool Care Pathway}

The quality of care given to patients with cancer at the end of life is suboptimal. In Italy, a third of patients with terminal cancer die in hospital. This situation prompted Massimo Costantini and colleagues to assess the effectiveness of the Italian version of the Liverpool Care Pathway (LCP-I).

As Costantini explains, "we decided to do this project after a number of discussions with physicians and nurses of Italian hospitals who were unsatisfied with the care received by patients with cancer at the end of their lives."

To carry out a randomized comparison, a cluster trial was undertaken in which 16 hospitals were randomly assigned to implement the LCP-I programme or standard health-care practice, and 308 patients who died from cancer were assessed in the 6 months after the completion of the intervention.

There was no significant difference in the overall quality of care (primary end point) between those wards following the LCP-I approach and control wards. Of the secondary outcomes that were assessed, two showed an improvement as a result of following the LCP-I programme-that of respect, dignity and kindness, and overall control of breathlessness.

Although no significant difference in overall quality of care was found for those receiving LCP-I, a small improvement was observed. "The LCP-I may have the potential to close the gap between hospice care and hospital care. While the results of this trial should be interpreted with caution, this is a robust trial and the study findings should be used to inform strategies to care for dying patients," says Constantini. Further research is needed to determine which fundamental components of the LCP-I might be beneficial. Costantini and collaborators are working on the next steps to establish such an improvement.

\section{Lisa Hutchinson}

Original article Costantini, M. et al. Liverpool Care Pathway for patients with cancer in hospital: a cluster randomised trial. Lancet doi:10.1016/S0140-6736(13)61725-0 